ESL students' perceptions of mobile applications for discipline-specific vocabulary acquisition for academic purposes

\title{
Lucas Kohnke
}

Adrian Ting

The Hong Kong Polytechnic University, Hong Kong

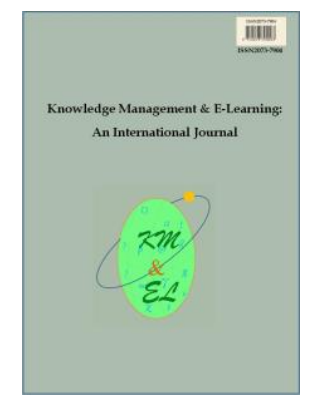

Knowledge Management \& E-Learning: An International Journal (KM\&EL) ISSN 2073-7904

Recommended citation:

Kohnke, L., \& Ting, A. (2021). ESL students' perceptions of mobile applications for discipline-specific vocabulary acquisition for academic purposes. Knowledge Management \& E-Learning, 13(1), 102-117. https://doi.org/10.34105/j.kmel.2021.13.006 


\title{
ESL students' perceptions of mobile applications for discipline-specific vocabulary acquisition for academic purposes
}

\author{
Lucas Kohnke* \\ English Language Centre \\ Faculty of Humanities \\ The Hong Kong Polytechnic University, Hong Kong \\ E-mail: lucas.kohnke@polyu.edu.hk
}

\author{
Adrian Ting \\ English Language Centre \\ Faculty of Humanities \\ The Hong Kong Polytechnic University, Hong Kong \\ E-mail: adrian.ting@polyu.edu.hk
}

*Corresponding author

\begin{abstract}
Undergraduates are often advised to expand their discipline-specific vocabulary in order to cope with the rigor of reading and writing academic texts. For the students studying in English-medium settings, it is in their interest to acquire vocabulary in their disciplines as this is vital to academic success. While students recognize the importance of discipline-specific vocabulary acquisition, they are typically unable to do so due to their heavy work on learning and assessment tasks. With the advent of mobile technology, one way to address this problem is to create vocabulary apps. To meet this need, this paper presents a gamified, discipline-specific vocabulary learning app Booksvs Brains@PolyU. It was developed to help busy undergraduates to build a repertoire of the vocabulary across seven disciplines. We explored students' perceptions and evaluation of this tool via interviews with sixteen student participants of the project. The results show that students found the tool useful and motivating, indicating the effectiveness of the app in helping busy undergraduates to build and expand their knowledge of discipline-specific vocabulary.
\end{abstract}

Keywords: Mobile-assisted language learning; Academic vocabulary; Vocabulary; Applications; Discipline-specific; EAP; ESL; Books vs Brains@PolyU

Biographical notes: Dr Kohnke is a Teaching Fellow in the English Language Centre at The Hong Kong Polytechnic University. Dr Kohnke has published articles in TESOL Journal, Journal of Education for Teaching, RELC Journal as well as other leading journals. His research interests include technologysupported teaching and learning, professional development using information communication technologies, and EAP/ESP course design.

Adrian Ting is an Instructor in the English Language Centre at The Hong Kong Polytechnic University. Mr. Ting has taught EAP and ESP courses to university students since 2003. His research interests include educational technology, 
EAP, ESP, and disciplinary writing. He has publications in the area of technology integration in ESL.

\section{Introduction}

Learning vocabulary constitute an essential aspect of studying a second or foreign language, including English for Academic Purposes (EAP). English as a second language (ESL) students at universities often find the process of understanding and acquiring the academic vocabulary for their chosen disciplines to be particularly challenging (Li \& Pemberton, 1994; Malmström, Pecorari, \& Shaw, 2018). Though academic vocabulary by definition appears frequently in academic texts, these terms are "not likely to be glossed by the content teachers" (Flowerdew, 1993, p. 236; Coxhead, 2000). Insufficient knowledge of vocabulary has often been identified as the greatest impediment to language students' academic success (Gardner \& Davis, 2014; Masrai \& Milton, 2018; Lesaux et al., 2014; Nation, 2001). Students' need to bolster their academic vocabularies has led to the creation of academic word lists, such as West's (1953) General Service List of 2,000-word families and Coxhead's (2000) Academic Word List of 570-word families, some of which are discipline-specific, such as Lei and Lin's (2016) medical word list and Liu and Han's (2015) environmental word list.

Generally, students who enter an English-medium university in Hong Kong realize that their English proficiency - particularly their knowledge, understanding, and range of vocabulary - is insufficient to support success in their academic studies (Evans \& Morrison, 2018; Morrison \& Evans, 2018). To compensate for this shortcoming, universities have made available learning and teaching resources to help ESL students transition from secondary education to university studies. Information technology has transformed English-language teaching and learning (Godwin-Jones, 2017). Thus, today's learners are highly mobile (Read \& Barcena, 2016) and are accustomed - and, indeed, prefer - to access language-learning materials digitally (Healey, 2018). Consequently, language educators have been designing, developing, and seeking out authentic digital materials to assist students in expanding their vocabularies (GodwinJones, 2017; Kohnke, Zou, \& Zhang, 2019; Kohnke, Zou, \& Zhang, 2020).

In recent years, mobile applications (apps) have shown the potential to enhance and transform language learning (Chen, Liu, \& Huang, 2019; Fu, 2018). In particular, apps that make it possible for learners to acquire vocabulary anywhere and anytime have attracted interest, as evidenced by discussions in the scholarship on mobile-assisted language learning (MALL; e.g., Burston, 2015; Stockwell, 2007). The acquisition of vocabulary by EAP learners, nevertheless, continues to be problematic (Shadiev, Hwang, \& Huang, 2017). Owing to the importance of developing discipline-specific vocabularies for these students - and to Chinese students' preference for utilizing apps in their language learning - this qualitative study explored the experiences and perspectives of students who were taking part in an academic mentoring program using a disciplinespecific vocabulary app, Books vs Brains@PolyU, that was developed in-house for this purpose. The study was designed to answer two main questions:

RQ1: What types of apps do students prefer to use to facilitate discipline-specific language learning?

RQ2: What are the factors that may influence students' use and perceptions of the BooksvsBrains@PolyU vocabularyapp? 


\section{Literature review}

In Hong Kong and elsewhere, the use of synchronous and asynchronous technology has become a fundamental aspect of teaching and learning in higher education institutions (Kukulska-Hulme, Lee, \& Norris, 2017). In tandem with this development has been an increase in research into the use of MALL in second-language acquisition to inform language studies more broadly (Loewen et al., 2019; Shadiev et al., 2017). For example, MALL provides new constructive opportunities for ESL learners to access content and enables teachers to supplement in-class instruction with an additional resource for authentic language learning that, again, can be accessed anytime and anywhere (Reinders \& Benson, 2017; Zou, Xie, \& Wang, 2018). In such ways, MALL supports students' autonomous development of English-language proficiency.

As mentioned, at universities, students must acquire a good grasp of a large number of discipline-specific words to succeed academically, though these words are seldom explained in textbooks. Indeed, the lack of familiarity with academic vocabulary has been identified as the greatest obstacle for many ESL learners (Moini \& Islamizadeh, 2016). Thus, teachers frequently consider it among their primary tasks to support students' development of discipline-specific vocabularies (Hyland \& Tse, 2007). In EAP classes, teachers tend to focus on words that appear in the target text, thereby limiting the scope of the vocabulary to which students are exposed (Pojanapunya, 2019).

Also as mentioned, established academic word lists can expand students' lexical range. Two of the more frequently used such lists are Coxhead's (2000) aforementioned Academic Word List (AWL) and the Academic Vocabulary List (AVL) by Gardner and Davies (2014). These two lists especially have had a considerable positive impact on EAP teaching and learning. However, Hyland and Tse (2007) argued that such general academic word lists are of limited usefulness owing to the wide variation in vocabulary across disciplines. Therefore, discipline-specific vocabulary lists that target ESL learners' specific academic goals have also been developed. Examples included the aforementioned medical word list by Lei and Lin (2016), Liu and Han's (2015) environmental word list, as well as Hsu's (2011) business word list and Yang's (2015) nursing word list. The potential benefits of using such lists include the establishment of vocabulary-learning goals and the assessment of vocabulary knowledge and growth. Again, however, each discipline-and even sub-discipline-often has a distinctive vocabulary (Durrant, 2014; Hyland \& Tse 2007).

EAP learners, then, should not be regarded as a homogeneous group but rather as individual students who tend to have a range of vocabulary needs (Coxhead, 2019; Dang, Coxhead, \& Webb, 2017). In their university-level studies, EAP students have only a limited time to develop their discipline-specific vocabulary and should "focus on specific, purposeful uses of language" (Hyland, 2016, p. 17). To maximize the use of this limited time for acquiring vocabulary in today's learning environment, it is important to understand individual students' preferences regarding the use of technology generally and in particular their perceptions of the effectiveness of MALL resources in facilitating language acquisition.

In Hong Kong and mainland China, apps have become tools of choice for, and indeed indispensable to, ESL students because they enable learners to access learning materials and to develop their proficiency independently (Ma, 2017). Consequently, several studies have investigated the use of apps to provide innovative forms of support for ESL learners, especially in terms of building vocabulary (e.g., Burston, 2015; Lin \& Lin, 2019). Some of these studies to date have focused on commercial apps, such as Babbel, Duolingo, and Rosetta Stone, and have contextualized learners' English language 
proficiency based on their educational levels and ages using quantitative, qualitative, or mixed-method approaches (Chen et al., 2019).

EAP students at universities in Hong Kong, then, frequently utilize dictionary apps to facilitate vocabulary acquisition (Ma, 2019) and to manage, learn about, and practice unfamiliar words (Dang \& Webb, 2014). Levy and Steel (2015) found that students had a preference for dictionary apps over traditional tools because of their portability and accessibility the time-savings. Also, several of the dictionary apps include multiple supporting functions, such as explanations, pictures, videos, and links to forums where users can discuss language-related issues.

Mason and Zhang (2017) reported that $95 \%$ of the Chinese EAP learners in their study used mobile apps autonomously and corroborated their positive impact on the learners' progress and development. This important finding demonstrates that students can use language apps independently and that apps have the potential to motivate and engage ESL learners specifically (Godwin-Jones, 2017). Liu, Zheng, and Chen (2019) identified three main motivations for Chinese students' use of dictionary apps in their academic studies: interest in learning new English words, functionality, and the perception that usage improved their performance on English-language assessments. Further, research conducted at a university in Hong Kong found that students were, indeed, keen to expand the range of their vocabulary both incidentally and deliberately outside the classroom (Ma, 2017; Kohnke, 2020; Zou, Wang, Xie, \& Kohnke, 2018). However, the use of apps for this purpose remains under-researched, especially when it comes to MALL and the development of discipline-specific vocabulary by EAP students in higher education.

The widespread growth in the use of ESL apps has led to the development of several frameworks for evaluating them. These frameworks take into account such factors as personalization, relevance, feedback, visible progress indicators, and usability in assessing an app's efficacy (Sweeney \& Moore, 2012). Previous research has identified among the advantages of language learning apps fitness for purpose, clear instructions, opportunities for individual feedback, and opportunities to practice multiple skills and established that learners prefer basic functions over advanced ones (Winestock \& Jeong, 2014). This result warrants further exploration so as to identify more precisely the functions that enhance students' language-learning experience and progress.

While the results of the research conducted thus far appear to have been positive, some studies have highlighted shortcomings of vocabulary apps as language-learning tools. Rosell-Aguilar (2016, 2018), for example, reported criticism of language learning apps including dissatisfaction with their focus on receptive skills, a lack of intuitiveness, and insufficient feedback and examples. The development of apps should be pedagogically-driven (Colpaert, 2006) and should not seek to replicate existing forms of technology if they are to motivate and engage learners. Consequently, it is important to understand the features of discipline-specific apps that language students' value when learning new vocabulary.

\section{Method}

The aim of this explorative, interpretive, qualitative study was to assess the perceptions of EAP students at an EMI university in Hong Kong regarding the use of an in-house app, Booksvs Brains@PolyU, for discipline-specific vocabulary learning. The study employed an interpretive approach to reveal the full complexity of the issues involved 
and provide insights in the form of information-rich responses that reflect the lived experiences of the participants as accurately as possible (Geertz, 1973).

\subsection{Learning tool/app}

The lead author developed a gamified, discipline-specific vocabulary learning app, Books vs Brains@PolyU, to engage and motivate students and expand their receptive vocabularies. The design of the app targeted university students in need of exposure to a wide range of words to improve and expand their knowledge of English vocabulary related to their university studies. Therefore, the forms and meanings of words were key components of the app's design (Nation, 2001). Previous vocabulary-acquisition app designs have highlighted spelling, the meanings of words, and pronunciation as the three essential components of learning a language (Ehri \& Rosenthal, 2007). Further, the interface was designed to be intuitive with respect to the effort necessary to start using the app, thereby maximizing the time that students would be able to devote to vocabulary acquisition (Godwin-Jones, 2017).

Learners can use Books vs Brains@PolyU to practice their knowledge and understanding of discipline-specific vocabulary. To do so, they draw on seven areas of learning, which are listed in the app as "disciplines/fields": Textiles and Clothing, School of Design, Faculty of Business, School of Nursing, Faculty of Engineering, Tourism Management, and Hotel Management (see Fig. 1).
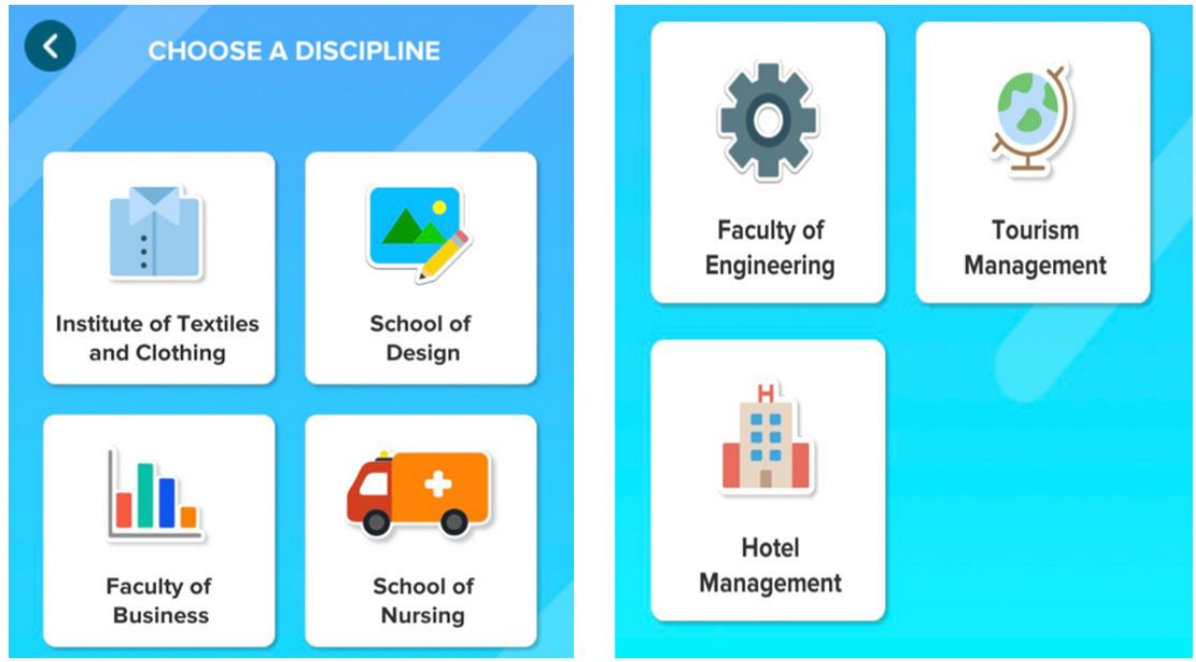

Fig. 1. Seven disciplines

Each discipline consists of four sub-levels-beginner, elementary, intermediate, and advanced - and focuses on 15 key questions or words at each level. An accumulation of 8 correct answers is required for progression to the next stage. If the player is stuck on a particular question, she has the option to either skip to the next one by pressing "Skip", or to receive a clue where two to three letters of the word appear upon clicking "Hint". A maximum of two hints are allowed for each word (see Fig. 2).

At the end of each level, the app displays the student's correct and incorrect responses to prompt words accompanied by the definitions and pronunciation details for each of these words (see Fig. 3). This vocabulary list can be downloaded on users' 
mobile devices to create academic and discipline-specific vocabulary banks. This feature is important because it helps students to learn from their mistakes and makes it easy for them to access vocabulary anytime and anywhere and thereby to consolidate their learning.
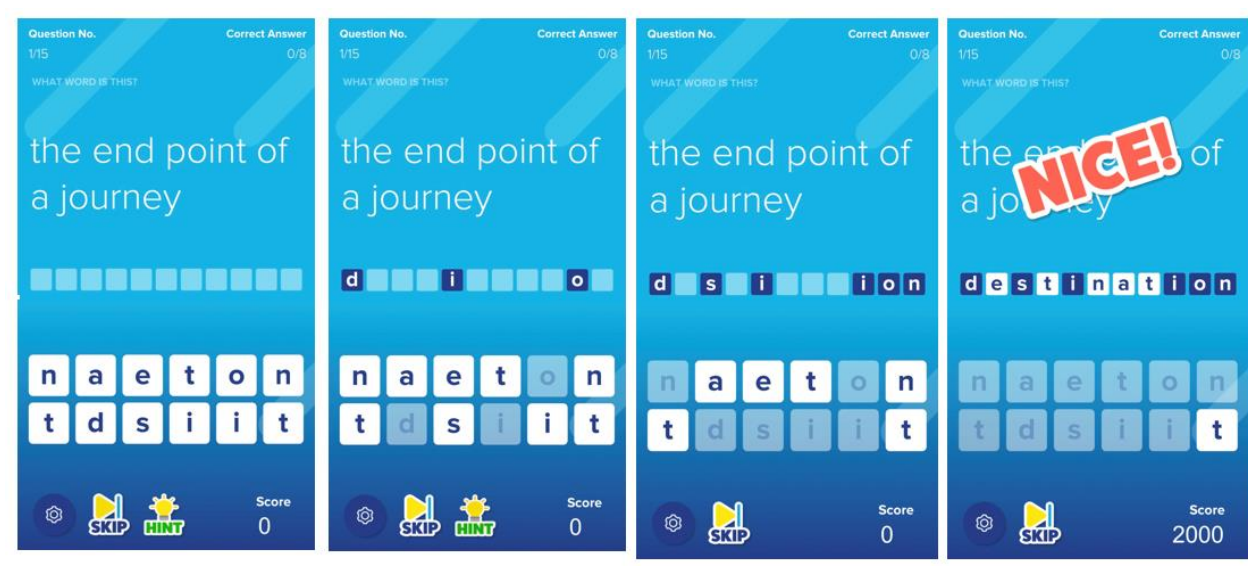

Fig. 2. Game mode

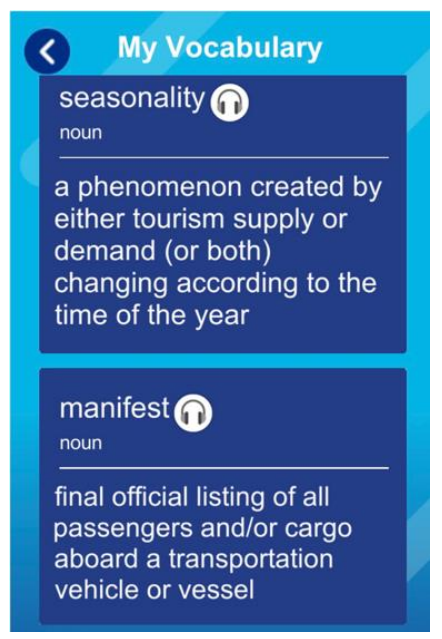

Fig. 3. Word definition and pronunciation

Further, each word is tailored to the particular difficulty level at which a student is studying and presented in a range of linguistic contexts that illustrate various aspects of speech in several sample sentences. Another game-based component that motivates users to continue playing is the option to post and share their scores on the digital leaderboard hosted on Facebook (see Fig. 4). These features augment students' efforts to increase and broaden their English vocabulary and their overall understanding of the language.

In seeking to meet the needs of university students in Hong Kong more effectively, we have found that existing word lists such as the aforementioned AWL and AVL are simply too wide-ranging to be suitable for students' particular learning needs. Consequently, the lead author established seven discipline-specific lists in conjunction with the seven disciplines/fields listed above consisting of words regarded as 
indispensable to each discipline. For this purpose, the lead author met with representatives from each of the departments corresponding to the seven disciplines and worked with them to identify which specific words ought to be included in the word lists. We then divided these words into the four categories of users from beginner to advanced. In this way, EAP learners' individual needs and abilities determined the content of each level of the app in a manner pitched appropriately to their studies.
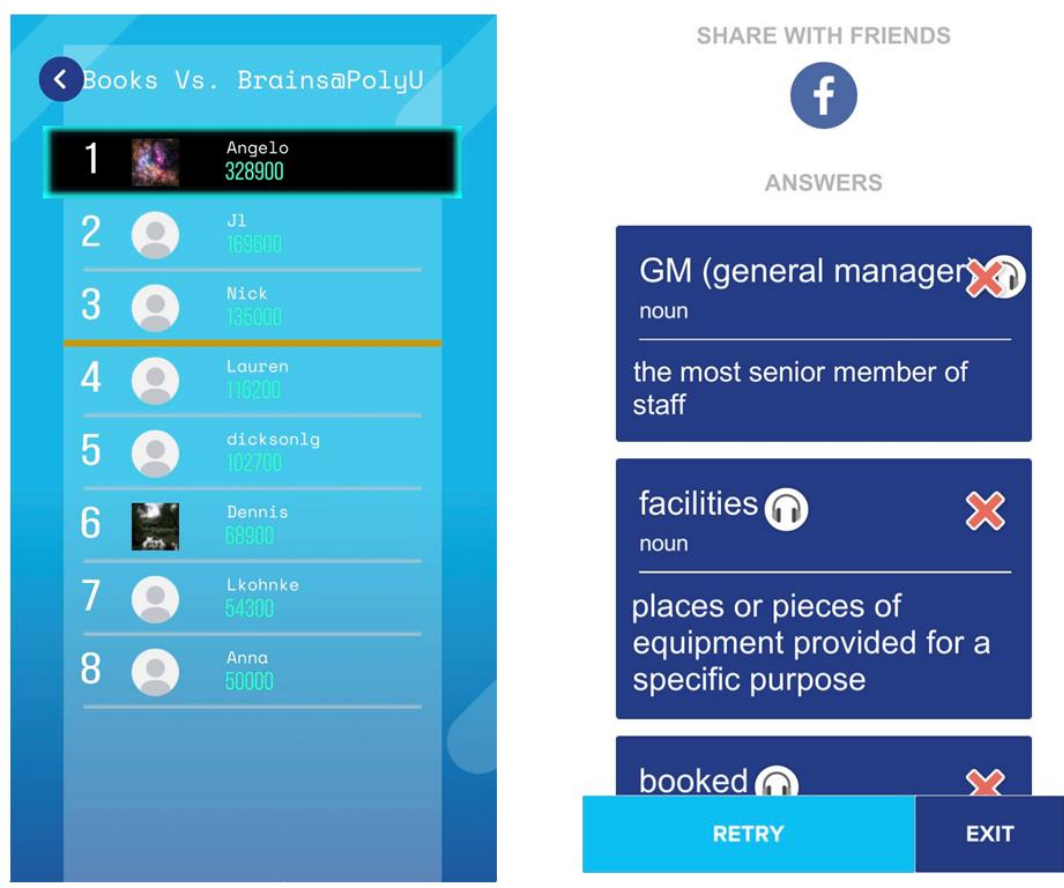

Fig. 4. Leaderboard and Facebook

\subsection{Participants}

The selection of participants for the focus groups followed the convenience sampling method which "involves choosing the nearest individuals to serve as respondents" (Cohen, Manion, \& Morrison, 2018, p. 218). An email invitation was sent to all 94 participants in a student-teacher mentoring program, and 16 first-year students volunteered to participate. The participants were originally from mainland China and Hong Kong, for whom English was either a foreign or second language, as is the case with the overall university population in Hong Kong. They included 9 female and 7 male either 18 or 19 years of age and together represented the seven disciplines represented in the app. They thus formed a representative sample capable of providing a holistic overview of students' perspectives. Since they had already volunteered to participate in the mentoring scheme, their motivations for studying and improving the quality of their English presumably differed somewhat from those of other university students. All of the participants gave their written consent and received an information sheet outlining the purpose and procedure for the research. 


\subsection{Data collection and analysis}

The 16 participants used the app for 10 weeks outside the classroom. Their usage data were then collected during four focus groups conducted in English that lasted from 34 to 47 minutes. The interview guide developed for the focus groups concerned the participants' experiences using the app for language-learning, aspects of it that they had found particularly useful, and their views of its efficacy in supporting language acquisition and university studies in their specific discipline. Focus group data are particularly useful for studies that strive to understand attitudes, opinions, and experiences of research subjects in a particular context (Ravitch \& Carl, 2016).

The focus-group sessions were audio-recorded and transcribed verbatim. Two rounds of member-checking served to establish the trustworthiness of the data (Merriam, 2017). First, each participant received a copy of the transcript of his or her focus group for approval, and none suggested any revisions. The six-step framework of Braun and Clarke (2006) provided the framework for the analysis of the data and helped to yield a rich, detailed, and complex account of the results. According to these researchers, thematic forms of analysis are flexible and are guided by researchers' key ideas and viewpoints. The participants completed the second member check when they confirmed that the final results and discussion of the research presented to them accurately represented their experiences.

\section{Findings}

\subsection{RQ1: What types of apps do students prefer to use to facilitate discipline- specific language learning?}

\subsubsection{Dictionary apps: Quick and easy access}

The first research question served to establish a general benchmark for the participants' preferred type of discipline-specific app for improving their vocabulary. All of them expressed a preference for direct translation between their L1 (Chinese) and L2 (English) and vice versa as well as the capability to enter Chinese simplified or traditional characters and pinyin. The participants reported that they found dictionary apps invaluable in terms of saving time during such in-class activities as lectures and tutorials as well as for completing such out-of-class tasks as reading course material, textbooks, and journal articles. This finding is consistent with previous studies of Chinese students' strategies for learning languages (Liu et al., 2019). One participant in the present study (S3) commented that "during lectures, there are words I don't understand on the PowerPoint, and I need to look them up quickly. With the English-Chinese dictionary, I can do so in seconds." The participants found the ability to input Chinese characters helpful for broadening discipline-specific vocabulary. As two of them reported,

"I can use my finger to quickly write the Chinese character, and then I can see the English meaning with several examples. This helps me to understand the meaning, and I can click on words to obtain more explanations. " (S6)

"This function saves lots of time . . as I think in Chinese it is easier to get the meaning of words, and then I know how to use the words in my writing. " (S9)

Another participant said that dictionary apps were important in that they, 
"Give me a definition of words in my major conveniently and help me to write my assignments." (S11)

These three comments reflect the overall impression that dictionary apps can accelerate the pace of learning. The lexical comprehensiveness (inclusion of synonyms, antonyms, example sentences, slang, etc.) in particular increased these learners' engagement. The participants' assertions corroborated previous findings that a significant challenge for students in EAP programs is the limited time available to develop discipline-specific vocabulary (Hyland, 2016). Thus, ready access to English-Chinese translation tools helps such students to understand unfamiliar vocabulary that they encounter in professional journals more efficiently. Unsurprising, the participants in the present study described effective dictionary apps as being user-friendly. When probed further about their thoughts on the efficacy of using these apps to expand their disciplinespecific vocabularies, though, several of the interviewees expressed reservations such as the following:

"Examples included are often of general English, so sometimes I'm still not sure [of] the meaning of the word in my context ... it can be very technical but it [is] still quite helpful as a starting point." (S1)

"Not always suitable for my major, or, if they include the word plus example, [then it] is still too difficult, and I still can't understand. It can be really frustrating." (S12)

Again, dictionaries and word lists cannot meet all of these students' various needs (Durrant, 2014), and many commercial dictionary apps lack discipline-specific technical terms or have only a limited lexical repertoire. Learners need access to the particular definitions of words that are used frequently in their disciplines. Another shortcoming of dictionary apps is that students tend to trust them less than traditional hard copies in terms of accuracy and comprehensiveness (Levy \& Steel, 2015). Previous studies have identified both shortcomings of dictionary apps as well as such benefits as multimodal input and output (Ma, 2019). There thus appears to be a need to develop a vocabulary app that incorporates multimodality with a specific focus on EAP learners' vocabulary needs across multiple disciplines and contexts.

\subsubsection{Language-learning apps and motivation}

In the focus-group interviews, four of the participants mentioned other types of language apps in addition to dictionary apps that they had tried, including Duolingo and Busuu. While many of them seemed to consider these apps useful for improving their English, they said that they were rarely able to sustain the necessary motivation to persist in using them. Thus, two of the participants explained that,

"Initially, they are fun but, after a while, I feel it gets boring." (S1)

"It is hard to find good ones; I mean, there is [so] much available, and many are very complicated and others too simple." (S8)

Another participant related,

"I have tried various language channels on WeChat and YouKu, but I feel it takes too long to watch the videos, and I have to write down keywords [and] sentences so I can remember them later. It is just not very efficient, and there is so much. It is really hard to find the good stuff." (S6)

This assessment of other apps was echoed by another student: 
"When I play, I feel "ok, I'm learning," but then afterward I never use the words, so what's the point?" (S7)

Nevertheless, the participants' statements confirmed overall that apps can serve as initial motivators in the learning process (Godwin-Jones, 2017), the problem again being that they were unable to sustain their language-learning independently. Previous research has found that ESL learners preferred simplicity and, for this reason, tended to utilize dictionary apps rather than social media channels, online language games, or other kinds of apps (Ma, 2019; Levy \& Steel, 2015). As language learners generally in developed countries have abundant access to MALL tools and the support to learn a language anywhere and anytime (Healey, 2018; Reinders \& Benson, 2017), they seem to be increasingly selective and well-informed in their choice of the particular lexical tools that work well for them in their specific environments. As would be expected, learners tend to seek quality, user-friendly tools that offer multiple functions.

\section{2. $R Q 2$ : What are the factors that may influence students' use and perceptions of the Books vs Brains@ PolyU vocabulary app?}

\subsubsection{Playing time and app design}

The interviewees indicated that, during the 10-week period, they used the app several times a week and discussed the time that they spent using it, and certain words that they encountered on it with their fellow mentees. This collaborative effort seems to have rendered the learning process more social and motivating. Thus, one of the participants stated,

"Personally, I found it really good to discuss with friends about words and definitions from the app. It was like, even though we were using the app, we could still discuss and learn." (S16)

Another participant described the points system as motivational: "It's fun and makes me wanna play longer to score higher than my friends" (S12). The interviewees consistently mentioned the gamified nature of the app as a key motivating factor-and, indeed, gamification seems to stimulate today's learners more than other teaching resources (Chen et al., 2019).

The participants also highlighted the intuitive capabilities of the app, with comments such as "Actually, it is very simple, so no instructions are needed" (S9) and "It is not fancy but very practical" (S2). The simplicity of the app's design contributed to its usability. As another participant mentioned,

"There are very few functions to handle... I see the explanation of the word and how many letters the word should have, plus, I can click on "Hint" to see a couple of letters in the definition, and then just drag the letters." (S5)

All of the participants gave positive feedback about the convenience of the user experience, but a few found that the interface design, though intuitive and easy to use, could be improved. One of them mentioned,

"Not much happening in the app; it would be better if it was more exciting, moving parts with short animations. Yes, more moving parts." (S8)

This is a fair criticism of the app, as more effort could have been made to integrate features that allow users to watch short animations and videos so that it would 
be more exciting for learners to complete each task. Various features of the design were mentioned during the focus groups as having enhanced the user experience, including the scoring system, hints, and pronunciation guidance. In any case, as the aforementioned comments demonstrate, the participants persevered in using the app. Several stressed that the app's design features had helped them to acquire new discipline-specific vocabulary.

\subsubsection{Levels of difficulty and understanding of definitions and examples}

The participants stated that the progression from level to level was helpful, and most of them agreed that the discipline-specific words were relevant to and useful for their studies. In particular, they liked the clear definition provided for each word as one said:

"The definition was shown in simple words which helped." (S10)

"Yes, the words describing the ideas were good. Most of them used very general .

. English, and this helped me to understand the word later." (S9)

Others found that being able to download the complete list of the words together with the pronunciation guidance on their phones when they had reached the end of each level was especially useful in terms of developing their discipline-specific vocabulary and motivating them to keep playing and, later, to continue using the app.

"I really liked having access to all [of] the words and being able to listen and [to]

try to pronounce them without having to play the game again." (S11)

"Explanations made it easier to understand the concept." (S16)

A few of the participants found that some of the words were too challenging overly long and complicated-which made it difficult for them to proceed to the next level. One stated, "I remember [that] one word was over 10 letters" (S15). Thus, while most of the participants believed that the definitions and examples provided in the app were appropriately pitched to their particular language level, this comment draws attention to the fact that their language levels differed. The differences among learners should, therefore, receive more attention in the development of apps. In light of research indicating that the nuances of English words are particularly puzzling for ESL learners (Crib \& Wang, 2019), it is clearly important to consider carefully the selection of both words and examples while developing vocabulary apps and word lists.

\subsubsection{Vocabulary learning and university studies}

The participants then described the mobile vocabulary app as useful (for example, S7). Though the students in the mentorship program were not required to use the mobile app for their coursework, several did so and were pleased with the results, as show in the following comments.

\footnotetext{
"Many of the words appeared in our readings and, later, I tried to incorporate them in my assignments." (S4)

"Throughout my introductory course, I kept looking at the words in the word list and listening to the pronunciation. And after a while, I became quite confident in using them during the tutorials. I want to say that some words I have never used, but I'm just a first-year student." (S13)
}

These are very pertinent insights, as the purpose of the app is to help students to develop discipline-specific vocabularies that will, in turn, help them with their academic studies. These comments are encouraging since they show that the participants went beyond the expected receptive vocabulary learning and attempted-and, at least to some 
extent, were successful in doing so-to learn to use appropriate and sophisticated vocabulary in their academic work.

One of the participants (S8) emphasized that EAP students find it difficult to understand discipline-specific words since they rarely encounter such words outside the context of the coursework for their majors. By using the Books vs Brains@PolyU app, students can learn the meanings of discipline-specific words through exposure to their use in simple sentences and their definitions. Several participants stated that the simple and plain English of the app provided a good starting point for acquiring specialized vocabulary. Other sentiments expressed repeatedly in the interviews included appreciation for the opportunity to repeat the levels and for the availability of new words. Two of the participants (S10, S2) suggested that the app would be better if learners could choose the level at which they wanted to play at any given time rather than being compelled to move from the beginner to the advanced level sequentially. One (S10) suggested that this improvement would make the app more challenging because students could preview the more difficult levels to see and be motivated by what is coming next.

\section{Conclusion}

The aim of this study was to gain an understanding of and to offer further insights into the use of a MALL mobile app by university students in Hong Kong to develop discipline-specific vocabulary. The findings presented here suggest that students at tertiary institutions in Hong Kong have been making increasing use of vocabulary apps to facilitate their understanding of subject-specific lectures and course readings. The participants in the study stated that dictionary apps facilitated access to direct translations of words, but several of them had found that such apps often contained only limited discipline-specific vocabulary. They responded favourably to the custom-built Books vs Brains@PolyU app, indicating that it had met their needs for building and expanding their knowledge of discipline-specific vocabulary. These results highlight the importance and potential of mobile apps for helping EAP learners of various abilities to succeed academically in English-medium universities and, specifically, the emancipatory educational effects of Books vs Brains@PolyU in terms of facilitating discipline-specific vocabulary acquisition.

The findings are promising overall, thought the study was relatively small in scale and did not measure the efficacy of the app itself. Instead, the focus was on students' strategies for using MALL and their perceptions of the in-house app. Future studies can build on these findings by collecting further data on students' progress with the written and spoken aspects of language using paired pre- and post-tests and on their levels of motivation over longer periods. Also, when developing a custom app for studies such as this one, it is important that researchers understand the technical constraints and intricacies involved, the limitations of the hardware (especially when designing such features as point systems, storing and downloading vocabulary lists, graphics, and social media integration), and the general and specific academic purposes for which English is used in various fields.

The development of discipline-specific vocabulary is vital for EAP students to succeed in their academic studies associated with their majors. Because students have limited time and opportunities to acquire such vocabulary, language apps can play an important role in facilitating independent learning by providing personalized and immediate feedback. The functionality of vocabulary apps can improve significantly 
when teachers share their strategies and resources for vocabulary acquisition with app developers and with one another.

\section{Author Statement}

The authors declare that they have no conflict of interest.

\section{ORCIID}

Lucas Kohnke (iD https://orcid.org/0000-0001-6717-5719

Adrian Ting (iD https://orcid.org/0000-0002-1354-9364

\section{References}

Braun, V., \& Clarke, V. (2006). Using thematic analysis in psychology. Qualitative Research in Psychology, 3(2), 77-101.

Burston, J. (2015). Twenty years of MALL project implementation: A meta-analysis of learning outcomes. ReCALL, 27, 4-20.

Chen, C. M., Liu, H., \& Huang, H. B. (2019). Effects of a mobile game-based English vocabulary learning app on learners' perception and learning performance: A case study of Taiwanese EFL learners. ReCALL, 31(2), 170-188.

Cohen, L., Manion, L., \& Morrison, K. (2018). Research methods in education (8th ed.). Abingdon, Oxon: Routledge.

Colpaert, J. (2006). Pedagogy-driven design for online language teaching and learning. CALICO Journal, 23(3), 477-497.

Coxhead, A. (2000). A new academic wordlist. TESOL Quarterly 34(2), 213-238.

Coxhead, A. (2019). Working with multi-word units in ESP/EAP. In E. Hinkel (Ed.). Teaching Essential Units of Language: Beyond Single-Word Vocabulary (pp. 36-54). New York, NY: Routledge.

Crib, M. V., \& Wang, X. (2019). Making academic vocabulary count through strategic deployment in oral presentations by Chinese students of English. Language Learning Journal. doi: 10.1080/09571736.2019.1566396

Dang, T. N. Y., Coxhead, A., \& Webb, S. (2017). The academic spoken word list. Language Learning, 67(4), 959-997.

Dang, T. N. Y., \& Webb, S. (2014). The lexical profile of academic spoken English. English for Specific Purposes, 33, 66-77.

Durrant, P. (2014). Discipline and level specificity in university students' written vocabulary. Applied Linguistics, 35(5), 328-356.

Ehri, L. C., \& Rosenthal, J. (2007). Spellings of words: A neglected facilitator of vocabulary learning. Journal of Literacy Research, 39(4), 389-409.

Evans, S., \& Morrison, B. (2018). Adjusting to higher education in Hong Kong: The influence of school medium of instruction. International Journal of Bilingual Education and Bilingualism, 21(8), 1016-1029.

Flowerdew, J. (1993). Concordancing as a tool in course design. System, 21(2), 231-244.

Fu, Q. K. (2018). Impacts of mobile technologies, systems and resources on language learning: A systematic review of selected journal publications from 2007-2016. Knowledge Management \& E-Learning, 10(4), 375-388.

Gardner, D., \& Davies, M. (2014). A new academic vocabulary list. Applied Linguistics, 35(3), 305-327. 
Geertz, C. (1973). The interpretation of cultures: Selected essays. New York, NY: Basic Books.

Godwin-Jones, R. (2017). Smartphones and language learning. Language Learning \& Technology, 21(2), 3-17.

Healey, D. (2018). Technology enhanced learning environments. In J. I. Liontas (Ed.), The TESOL Encyclopedia of English Language Teaching. Hoboken, NJ: Wiley. doi: 10.1002/9781118784235.eelt0437

Hsu, W. (2011). A business word list for prospective EFL business postgraduates. The Asian ESP Journal, 7(4), 63-99.

Hyland, K. (2016). General and specific EAP. In K. Hyland \& P. Shaw (Eds.), The Routledge Handbook of English for Academic Purposes (pp.17-29). Abingdon, Oxon, UK: Routledge.

Hyland, K., \& Tse, P. (2007). Is there an "Academic vocabulary'? TESOL Quarterly, 41(2), 235-253.

Kohnke, L. (2020). Exploring learner perception, experience and motivation of using a mobile app in L2 vocabulary acquisition. International Journal of Computer-Assisted Language Learning and Teaching, 10(1), 15-26.

Kohnke, L., Zou, D., \& Zhang, R. (2019). Using mobile vocabulary learning apps as aids to knowledge retention: Business vocabulary acquisition. The Journal of Asia TEFL, 16(2), 683-690.

Kohnke, L. Zou, D., \& Zhang, R. (2020). Exploring discipline-specific vocabulary retention in L2 through app design: Implications for higher education students. RELC Journal. doi: 10.1177/0033688219899740

Kukulska-Hulme, A., Lee, H., \& Norris, L. (2017). Mobile learning revolution: Implications for language pedagogy. In C. A. Chapelle \& S. Sauro (Eds.), The Handbook of Technology and Second Language Teaching and Learning (pp. 217233). Oxford, UK: Wiley \& Sons.

Lei, L., \& Lin, D. (2016). A new medical academic word list: A corpus-based study with enhanced methodology. Journal of English for Academic Purposes, 22, 42-53.

Lesaux, N. K., Kieffer, M. J., Kelley, J. G., \& Harries Russ, J. (2014). Effects of academic vocabulary instruction for linguistically diverse adolescents: Evidence from a randomized field trial. American Educational Research Journal, 51(6), 1159-1194.

Levy, M., \& Steel, C. (2015). Language learner perspectives on the functionality and use of electronic language dictionaries. ReCALL, 27(2), 177-196.

Li, E. S. L., \& Pemberton, R. (1994). An investigation of students' knowledge of academic and subtechnical vocabulary. In Proceedings of the joint Seminar on Carpus Linguistics and Lexicology (pp. 183-196). Hong Kong: Hong Kong University of Science and Technology.

Lin, J. J., \& Lin, H. (2019). Mobile-assisted ESL/EFL vocabulary learning: A systematic review and meta-analysis. Computer Assisted Language Learning, 32(8), 878-919.

Liu, J., \& Han, L. (2015). A corpus-based environmental academic word list building and its validity test. English for Specific Purposes, 39, 1-11.

Liu, X., Zheng, D., \& Chen, Y. (2019). Latent classes of smartphone dictionary users among Chinese EFL learners: A mixed-method inquiry into motivation for mobile assisted language learning. International Journal of Lexiography, 32(1), 68-91.

Loewen, S., Crowther, D., Isbell, D. R., Kim, K. M., Maloney, J., Miller, Z. F., \& Rawal, H. (2019). Mobile-assisted language learning: A Duolingo case study. ReCALL, 31(3), 293-311.

Ma, Q. (2017). A multi-case study of university students' language-learning experience mediated by mobile technologies: A socio-cultural perspective. Computer Assisted Language Learning, 30(3/4), 183-203. 
Ma, Q. (2019). University L2 learners' voices and experiences in making use of dictionary apps in mobile assisted language learning (MALL). International Journal of Computer-Assisted Language Learning and teaching, 9(4), 18-36.

Malmström, H., Pecorari, D., \& Shaw, P. (2018). Words for what? Contrasting university students' receptive and productive academic vocabulary needs. English for Specific Purposes, 50, 28-39.

Mason, A., \& Zhang, W. (2017). An exploration of the use of mobile applications to support the learning of Chinese characters employed by students of Chinese as a foreign language. In Q. Kan \& S. Bax (Eds.), Beyond the Language Classroom: Researching MOOCs and Other Innovations (pp. 99-112). Research-publishing.net.

Masrai, A., \& Milton, J. (2018). Measuring the contribution of academic and general vocabulary knowledge to learners' academic achievement. Journal of English for Academic Purposes, 31, 44-57.

Merriam, S. B. (2017). Qualitative research: A guide to design and implementation (2nd ed.), San Francisco, CA: Jossey-Bass.

Moini, R., \& Islamizadeh, Z. (2016). Do we need discipline-specific academic word lists? Linguistics academic word list (LAWL). Journal of Teaching Language Skills, 35(3), 65-90.

Morrison, B., \& Evans, S. (2018). Supporting non-native speaker student writers making the transition from school to an English-medium university. Language Learning in Higher Education, 8(1), 1-20.

Nation, I. S. P. (2001). Learning vocabulary in another language. Cambridge, UK: Cambridge University Press.

Ravitch, S. M., \& Carl, N. M. (2016). Qualitative research: Bridging the conceptual, theoretical, and methodological. Thousand Oaks, CA: Sage.

Read, T., \& Barcena, E. (2016). Metacognition as scaffolding for the development of listening comprehension in a social MALL app. RIED: Revista Iberoamericana de Educación a Distancia, 19(1), 103-120.

Reinders, H., \& Benson, P. (2017). Research agenda: Language learning beyond the classroom. Language Teaching, 50(4), 561-578.

Rosell-Aguilar, F. (2016). User evaluation of language learning mobile applications: A case study with learners of Spanish. In A. Palalas \& M. Ally (Eds.), The International Handbook of Mobile-Assisted Language Learning (pp. 454-581). Beijing, CN: China Central Radio \& TV University Press.

Rosell-Aguilar, F. (2018). Autonomous language learning through a mobile application: A user evaluation of the Busuu app. Computer Assisted Language Learning, 31(8), 854-881.

Shadiev, R., Hwang, W. Y., \& Huang, Y. M. (2017). Review of research on mobile language learning in authentic environments. Computer Assisted Language Learning, $30(3 / 4), 284-303$.

Stockwell, G. (2007). Vocabulary on the move: Investigating an intelligent mobile phone-based vocabulary tutor. Computer Assisted Language Learning, 20(4), 365383.

Sweeney, P., \& Moore, C. (2012) Mobile apps for learning vocabulary: Categories, evaluation and design criteria for teachers and developers. International Journal of Computer-Assisted Language Learning and Teaching, 2(4), 1-16.

Pojanapunya, P. (2019). Tailoring academic words to multidisciplinary EAP classes. TESOL Journal, 10(3): e00439.

West, M. (1953). A general service list of English words. London, UK: Longman, Green $\&$ Co.

Winestock, C., \& Jeong, Y. (2014). An analysis of the smartphone dictionary app market. Lexicography: Journal of ASIALEX, 1(1), 109-119. 
Yang, M. N. (2015). A nursing academic word list. English for Specific Purposes, 37, $27-38$.

Zou, D., Xie, H., \& Wang, F. L. (2018). Future trends and research issues of technologyenhanced language learning. A technological perspective. Knowledge Management \& E-Learning, 10(4), 426-440.

Zou, D., Wang, F. L., Xie, H., \& Kohnke, L. (2018). Game-based vocabulary learning in China and Hong Kong: Students' evaluation of different word learning apps. In Proceedings of the International Conference on Technology in Education (pp. 44-55). Springer. 